not fit the data. The best fit of the TOO1 and TOO2 flux data versus time was obtained with a power-law function $\left(\propto t^{-\alpha}\right)$ (see Fig. 3). The best-fit index is given by $\alpha=1.33_{-0.11}^{+0.13}\left(\chi^{2}\right.$ per d.o.f. $=0.7$ with 4 d.o.f.).

We have also compared the flux and the decay law found for 1SAX J0501.7 + 1146 with the fluxes measured with GRBM and WFC during the $\gamma$-ray burst and during the following minor pulses shown in Fig. 1. In Fig. 3 (top left), the dashed line shows the $2-10 \mathrm{keV}$ flux averaged over $100 \mathrm{~s}$ corresponding to the entire burst duration, whereas the solid horizontal line gives the average flux of the three minor pulses. Both fluxes are consistent with the backward extrapolation of the derived afterglow decay law. This strongly suggests that the X-ray emission detected soon after the GRB continuously evolves into the X-ray emission of the afterglow.

This result has an implication for the energetics of the event. The GRB fluence measured by GRBM in the $40-700 \mathrm{keV}$ band was $1.1 \times 10^{-5} \mathrm{erg} \mathrm{cm}^{-2}$. The X-ray fluence measured by WFC in the $2-10 \mathrm{keV}$ band was $\sim 1.2 \times 10^{-6} \mathrm{erg} \mathrm{cm}^{-2}$, that is $\sim 11 \%$ of the $\gamma$ ray fluence. If we assume that the three last pulses in Fig. 1 are part of the afterglow, by integrating the power law from $35 \mathrm{~s}$ to infinity we find, in the window $2-10 \mathrm{keV}$, a fluence which is $\sim 40 \%$ of the energy in the $\gamma$-ray burst itself in the band $40-700 \mathrm{keV}$. The X-ray afterglow is not only the low-energy tail of the GRB phenomenon but is also a significant channel of energy dissipation of the event on a completely different timescale.

The well-established power-law decay function of the GRB remnant flux, the consistency of its extrapolation with the X-ray flux at the time of the burst, and the energetic content in X-rays are the main results of our discovery. They will significantly affect models of GRBs and constrain their parameters. Indeed the fast detection of GRB970228, promptly communicated to the scientific community ${ }^{3,4}$, triggered both the Beppo-SAX NFI follow-up and observations in the radio ${ }^{19,20}$ and optical bands ${ }^{21-25}$. These observations lead to cogent limits to the radio emission and to the detection $^{5,26-30}$ of an optical transient, in a position consistent with that of 1SAX J0501.7 + 1146 that faded in a few days. We note, however, that a previous GRB detected by Beppo-SAX, GRB970111 $1^{31}$, had a $\gamma$-ray fluence about four times larger than GRB970228 and an undetectable X-ray emission 16 hours after the burst. No fading optical source was detected at a level of magnitude $B=23$ and $R=22.6$ (ref. 32 ).

The Beppo-SAX measurement, in addition to discovering a relevant delayed $\mathrm{X}$-ray emission, has thus provided the link missing for 25 years between the $\gamma$-ray phenomenology and the ultimate location capability of X-ray, optical and radio astronomy. We expect more detections of GRBs by Beppo-SAX GRBM/WFC, along with their follow-up observations. We hope that the existence of X-ray/ optical afterglows and their rapid detection will contribute to the unambiguous identification of the GRB sources.

Received 9 April; accepted 22 May 1997

1. Fishman, G. J. \& Meegan, C. A. Gamma ray bursts. Annu. Rev. Astron. Astrophys. 33, 415-458 (1995) 2. Boella, G. et al. Beppo SAX, the wide band mission for X-ray astronomy. Astron. Astrophys. Suppl. Ser. 122, 299-307 (1997).

Costa, E. et al. IAU Circ. No. 6572 (1997).

4. Costa, E. et al. IAU Circ. No. 6576 (1997)

5. van Paradijs, J. et al. Transient optical emission from the error box of the $\gamma$-ray burst of 28 February 1997. Nature 386, 686-688 (1997).

6. Frontera, F. et al. IAU Circ. No. 6637 (1997)

7. Vrba, F. Searches for gamma-ray bursts counterparts: current status and future prospects. 565-574 (AIP Proc. 384, Am. Inst. Phys., Huntsville, AL, 1996).

8. Piro, L., Scarsi, L. \& Butler, R. C. in X-Ray and EUV/FUV Spectroscopy and Polarimetry (ed. Fenischi, S.) 169-181 (SPIE 2517, San Diego, 1995).

9. Costa, E. et al. The gamma-ray burst monitor onboard SAX. Adv. Space Res. (submitted).

10. Frontera, F. et al. The high energy instruments PDS on-board the BeppoSAX X-ray astronomy satellite. Astron. Astrophys. Suppl. Ser. 122, 357-369 (1997).

11. Jager, R. et al. The Wide Field cameras onboard the BeppoSAX X-ray astronomy satellite. Astron Astrophys. Suppl. Ser. (submitted).

12. Parmar, A. et al. The low energy concentrator spectrometer on-board the BeppoSAXX-ray astronomy satellite. Astron. Astrophys. Suppl. Ser. 122, 309-326 (1997).

13. Boella, G. et al. The medium energy concentrator spectrometer on-board the BeppoSAX X-ray astronomy satellite. Astron. Astrophys. Suppl. Ser. 122, 327-340 (1997).

14. Manzo, G. et al. The high pressure gas scintillation proportional counter on-board the BeppoSAX X-ray astronomy satellite. Astron. Astrophys. Suppl. Ser. 122, 341-356 (1997)
15. Hurley, K. et al. 3rd Interplanetary Network localization, time history, fluence, and peak flux of the February 28, 1997 gamma-ray burst. Astrophys. J. Lett. (in the press).

16. Boller, T. et al. IAU Circ. No. 6580 (1997).

17. Warwick, R. \& Stewart, G. in Proc. 23rd ESLAB Symp. on Two-Topics in X-Ray Astronomy (eds Hunt, J. \& Batteick, B.) 727-731 (SP-296, ESA, Bologna, 1989).

18. Cline, T. L. et al. IAU Circ. No. 6593 (1997).

19. Galama, T. J. et al. IAU Circ. No. 6574 (1997)

20. Frail, D. A. et al. IAU Circ. No. 6576 (1997).

21. Metzger, R. M. et al. IAU Circ. No. 6588 (1997).

22. Pedersen, H. et al. IAU Circ. No. 6580 (1997).

23. Wagner, et al. IAU Circ. No. 6581 (1997).

24. Tonry, J. L. et al. IAU Circ. No. 6620 (1997).

25. Metzger, M. R. et al. IAU Circ. No. 6631 (1997).

26. Guarnieri, A. et al. Earliest detection of an optical transient following the gamma-ray burst GRB970228. Astron. Astrophys. Lett. (submitted).

27. Margon, B. et al. IAU Circ. No. 6618 (1997).

28. Pedichini, F. et al. IAU Circ. No. 6635 (1997).

29. Sahu, K. et al. IAU Circ. No. 6606 (1997).

30. Sahu, K. et al. IAU Circ. No. 6619 (1997).

31. Costa, E. et al. IAU Circ. No. 6533 (1997).

32. Castro-Tirado, A. et al. IAU Circ. No. 6598 (1997)

33. Georgentopoulos, I. et al. ASCA observations of deep ROSAT fields I: the nature of the X-ray source populations. Mon. Nat. R. Astron. Soc. (in the press).

34. Yoshida, A. et al. IAU Circ. No. 6593 (1997).

Acknowledgements. This research is supported by the Italian Space Agency (ASI) and Consiglio Nazionale Ricerche of Italy. Beppo-SAX is a joint program of ASI and the Netherlands Agency for Aerospace Programs (NIVR). We thank the staff at the Beppo-SAX Scientific Operation Centre and the Operation Control Centre for their contributions to the GRB research program.

Correspondence should be addressed to E.C. (e-mail: costa@saturn.ias.fra.cnr.it).

\section{A possible long-lived belt of objects between Uranus and Neptune}

\section{Matthew J. Holman}

Canadian Institute for Theoretical Astrophysics, McLennan Physical Laboratories, University of Toronto, 60 St George Street, Toronto, Ontario M5S 3H8, Canada

Recent discoveries of objects orbiting beyond Neptune ${ }^{1-5}$ have emphasized that our understanding of the distribution and dynamics of material in the outer Solar System is very incomplete. This trans-neptunian population-known as the Kuiper belt-is thought to act as a relatively stable reservoir of objects that could become short-period comets ${ }^{6-9}$, although there may be other regions of stability in the outer Solar System that could also supply such comets. Here I use numerical simulations to identify one such long-lived region between the orbits of Uranus and Neptune. I show that in the region 24-27 aU from the Sun, about 0.3 per cent of an initial population of small bodies moving on low-eccentricity, low-inclination orbits could survive for the age of the Solar System. The actual existence of this hypothetical belt is not precluded by currently available observational limits, and there could be as much as $\sim 5 \times 10^{-4}$ Earth masses of material populating this region-comparable to the mass of the asteroid belt between Mars and Jupiter.

Numerical investigations show there are few such regions in the outer Solar System where material could survive for the age of Solar System without being ejected through the gravitational influence of the giant planets ${ }^{7,10}$. In a recent study ${ }^{7}$, a few thousand test particles, starting from circular orbits in the plane of the Solar System from 5 to $50 \mathrm{AU}$ from the Sun, were numerically integrated for times up to $800 \mathrm{Myr}$. Later work extended this to $4.5 \mathrm{Gyr}$ for the regions between the outer planets and to $1 \mathrm{Gyr}$ beyond Neptune ${ }^{11}$. During the integrations, any test particle that entered the gravitational sphere of influence of a planet ${ }^{12}$ was removed from the simulation. Such test particles are typically ejected from the Solar System in another $10^{6}-10^{7} \mathrm{yr}$ (ref. 13). The results are shown in Fig. 1 . The green points mark the close encounter times as a function of semimajor axis; the red points indicate those test particles that survived the full 


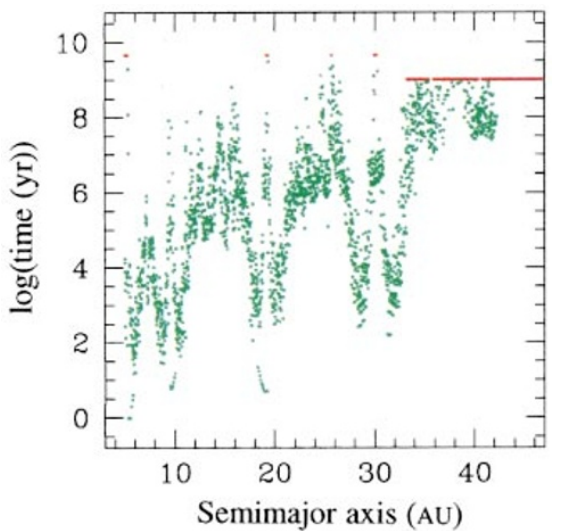

Figure 1 Time survived by each test particle as a function of initial semimajor axis. Green points, times at which the test particles encountered a planet; red points, those test particles which survived the full integration, 4.5 Gyr interior to Neptune and 1 Gyr exterior to Neptune. Beyond $\sim 43$ Au all the test particles survive the full integration. The scatter of points gives an idea of the spread of termination times for any given semimajor axis. The spikes at 5.2, 9.5, 19.2 and $30.1 \mathrm{AU}$, at the semimajor axes of the outer planets, correspond to test particles librating in Trojan or horseshoe-like orbits. A few test particles remain at locations of Jupiter, Uranus and Neptune. The absence of Saturn Trojans may not be significant as the test particles were not explicitly placed near the $L_{4}$ and $L_{5}$ Lagrange points of the outer planets. Between the planets nearly all of the test particles have been removed.

integration. Beyond Neptune (at $30.1 \mathrm{AU}$ ), the gravitational erosion in the Kuiper belt is apparent, yet regions stable on $10^{9}$-yr timescales can be clearly identified. Apart from a few test particles near the $\mathrm{L}_{4}$ and $L_{5}$ Lagrange points of the outer planets, nearly all of the test particles started in the outer planet region have been removed. Here I note that one solitary particle, at $25.7 \mathrm{AU}$, remains in the $24-27 \mathrm{AU}$ region after $4.5 \mathrm{Gyr}$.

To determine the significance of this single survivor, I integrated the orbits of several thousand additional test particles in the 24$27 \mathrm{AU}$ region. The initial semimajor axes of the test particles were distributed uniformly from 24 to $27 \mathrm{AU}$. The initial eccentricity and inclination ranges were $e_{0}=0.00-0.05$ and $i_{0}=0^{\circ}-10^{\circ}$, respectively. The orbits were integrated for times up to $4.5 \mathrm{Gyr}$. No test particle with $e_{0}>0.03$ or $i_{0}>3^{\circ}$ survived for more than $1 \mathrm{Gyr}$; no test particle with $e_{0}>0.01$ or $i_{0}>1^{\circ}$ survived for the full $4.5 \mathrm{Gyr}$. Including the single survivor from the initial simulation, five test particles out of 1,700 (or $0.3 \%$ ) with $e_{0} \leqslant 0.01$ and $i_{0} \leqslant 1^{\circ}$ survived 4.5 Gyr. Figure 2 shows the time survived as a function of initial semimajor axis for those test particles with $e_{0} \leqslant 0.01$ or $i_{0} \leqslant 1^{\circ}$ in the new simulation. Two regions, near $24.6 \mathrm{AU}$ and $25.6 \mathrm{AU}$, harbour the longest-lived test particles.

Figure 3 shows the number of test particles remaining in the 24$27 \mathrm{AU}$ region as a function of time. Assuming a power-law decay profile after $0.1 \mathrm{Gyr}$, a maximum-likelihood analysis yields an exponent of $-1.01 \pm 0.13$, in agreement with the $t^{-1}$ decay (where $t$ is the elapsed integration time) of objects with planetcrossing orbits ${ }^{14}$. The dashed line, with slope of -1 , is given for reference. Extrapolating from 236 test particles remaining at $0.1 \mathrm{Gyr}$ we would expect $5 \pm 2$ test particles at $4.5 \mathrm{Gyr}$, assuming $t^{-1}$ decay. The remaining test particles are not anomalies but the end result of the slow decay of an initially larger population. The survivors are not stable in the sense that they will remain indefinitely: their orbits are chaotic with Lyapunov times of $\sim 6 \times 10^{5} \mathrm{yr}$. Probably, the $t^{-1}$ decay profile will continue until all the test particles are removed.

Other processes may have significantly reduced the population of bodies between Uranus and Neptune. Collisions are thought to have eroded the population in the inner regions of the Kuiper belt ${ }^{15,16}$ and

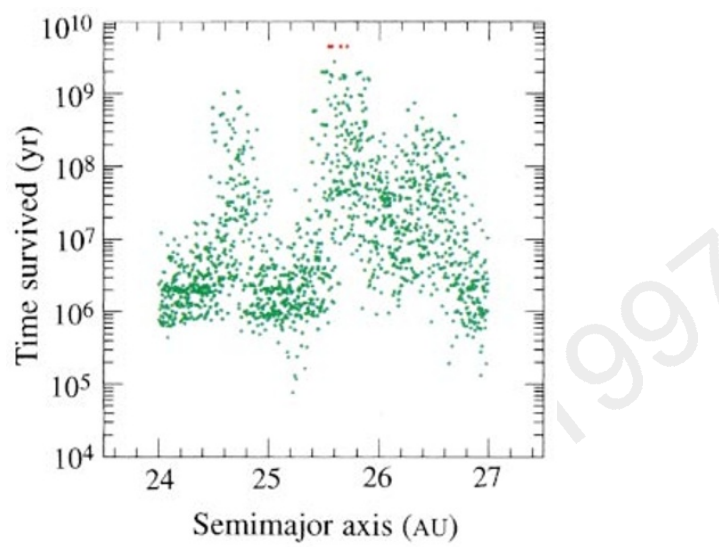

Figure 2 Results of additional simulations of the region between Uranus and Neptune. The test particles here had low initial eccentricity and inclination, $e_{0} \leqslant 0.01$ and $i_{0} \leqslant 1^{\circ}$. Again, the green points mark the times at which test particles encountered a planet. The red points indicate those test particles which survived the full 4.5-Gyr integration. The regions near 24.6 AU and 25.6 AU harbour the longest-lived members of the 24-27 Au region.

could have had a similar effect in the Uranus-Neptune region. Likewise, planet migration or the presence of large scattering bodies during the late stages of planet formation would tend to increase the typical eccentricity or inclination of the population, thereby reducing the fraction of material surviving to the present $t^{17-20}$. However, if we assume that that collisional processes removed a similar fraction of material from the Uranus-Neptune region as from the Kuiper belt, and if we assume that primordial material had low eccentricities and inclinations, then we can compare the number of objects in the Uranus-Neptune region to that in the Kuiper belt. The current number of bodies indigenous to the $24-27 \mathrm{AU}$ belt is roughly

$$
N_{\text {ind }} \propto \sigma_{25} A_{\text {ind }} f_{\text {ind }}
$$

where $\sigma_{25}$ is the initial mass surface density at $25 \mathrm{AU}, A_{\text {ind }}$ is the area of the $24-27 \mathrm{AU}$ belt, and $f_{\text {ind }}$ is the fraction of the population not removed by the gravitational influence of the giant planets. From the numerical integrations, $f_{\text {ind }} \approx 0.003$. The number of Kuiper-belt objects around $40 \mathrm{AU}$ is, correspondingly,

$$
N_{\mathrm{k}} \propto \sigma_{40} A_{\mathrm{k}} f_{\mathrm{k}}
$$

Here I adopt $f_{\mathrm{k}} \approx 0.4$ (refs 7, 21). Assuming $\sigma(r) \approx r^{-2}$ (ref. 22) (where $r$ is the heliocentric distance), and taking the 24-27 AU belt and the Kuiper belt to be annuli, $3 \mathrm{AU}$ and $10 \mathrm{AU}$ wide, centred on $25 \mathrm{AU}$ and $40 \mathrm{AU}$, respectively, then

$$
N_{\text {ind }} \approx\left(\frac{40}{25}\right)^{2}\left(\frac{25 \times 3}{40 \times 10}\right)\left(\frac{0.003}{0.4}\right) N_{\mathrm{k}}=4 \times 10^{-3} N_{\mathrm{k}}
$$

There are estimated ${ }^{4}$ to be $7 \times 10^{4}$ objects in the Kuiper belt with radii greater than $50 \mathrm{~km}$. Equation (3) implies that there are $\sim 300$ objects of similar size in the 24-27 AU belt.

This estimated population in the region does not violate available observations, despite assumptions that tend towards an overestimate. Two surveys in particular constrain the population in the 24$27 \mathrm{AU}$ region. The first survey, by $\mathrm{Kowal}^{23}$, examined 6,400 square degrees of sky to an R-band limiting magnitude $m_{R}=18.5$. The limiting magnitude has been adjusted for an estimated trailing loss 


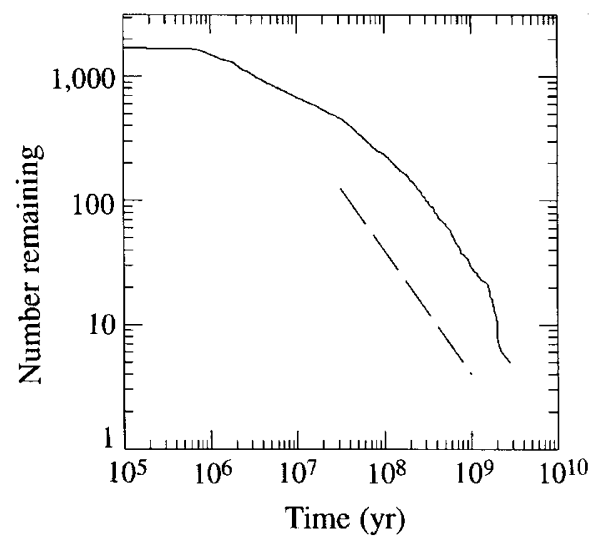

Figure 3 The solid line marks the number of test particles remaining as a function of integration time, for those particles started in the interval 24-27 AU. After 0.1 Gyr the profile approaches $t^{-1}$ decay. The dashed line, with a slope of -1 , is shown for reference.

of $\sim 1$ magnitude (ref. 4). Kowal's survey yielded 2060 Chiron, the first Centaur comet. The second survey, by Jewitt, Luu and Chen, combines two fainter searches of 1.2 and 3.9 square degrees near the ecliptic to $m_{R}=24.2$ that together yielded discoveries of 17 Kuiperbelt objects and two Centaurs (refs 1, 4). The Centaurs discovered to date have semimajor axes outside of 24-27 AU and/or high eccentricities: thus they are not indigenous members of the $24-27 \mathrm{AU}$ region. The observations can only provide an upper limit to the number of objects that could occupy this region and still reasonably escape detection. The apparent magnitude of an object is related to its absolute magnitude by

$$
m_{R}=H_{R}+5 \log _{10}(r \Delta)
$$

where $r$ is the heliocentric distance (in $\mathrm{AU}$ ) and $\Delta=r-1$ is the geocentric distance at opposition (also in $\mathrm{AU}$ ). For objects with $r=25 \mathrm{AU}$, the searches could detect objects with $H_{R}<4.6$ and $H_{R}<10.3$, respectively. Neglecting phase-angle effects, the radius, $z$, of an object (in kilometres) given its absolute magnitude $H_{R}$ is

$$
z=3.1 \times 10^{3}\left(\frac{0.03}{p_{R}}\right)^{1 / 2} 10^{-0.2 H_{R}}
$$

where $p_{R}$ is its R-band geometric albedo (ref. 2). Assuming $p_{R}=0.04, r=25 \mathrm{AU}$, and $\Delta=24 \mathrm{AU}$, Kowal's survey and the Jewitt-Luu-Chen survey could only detect objects with radii $z>320 \mathrm{~km}$ and $z>25 \mathrm{~km}$, respectively. At $99.9 \%$ confidence there can be at most seven objects with $z>320 \mathrm{~km}$, and 2,900 objects with $z>25 \mathrm{~km}$ in a $6^{\circ}$ wide ecliptic band. This assumes all objects with inclinations greater than $3^{\circ}$ have been removed dynamically.

The relative importance of these two constraints depends on the size distribution in the region. The number of objects with radii greater than $z$ can be approximated by a power law such that

$$
N(>z) \propto z^{1-q}
$$

where $3 \leqslant q \leqslant 5$ (from studies of Kuiper-belt objects and shortperiod comets $\left.{ }^{2,4,9,24}\right)$. For $q=3$, Kowal's survey imposes the stronger constraint, $N(>50 \mathrm{~km})<300$. For $q=5$, the Jewitt-Luu-Chen survey is more restrictive, $N(>50 \mathrm{~km})<180$. Both constraints are comparable to the estimate of $N(>50 \mathrm{~km}) \approx 300$ from equation (3). Assuming an average density of $\rho=1,000 \mathrm{~kg} \mathrm{~m}^{-3}$ and arbitrarily considering only objects with radii between 10 and $500 \mathrm{~km}$, the observational upper limits to the mass in the 24-27 AU region are $3 \times 10^{21} \mathrm{~kg}\left(5 \times 10^{-4}\right.$ Earth masses $)$ for $q=3$ and $2 \times 10^{21} \mathrm{~kg}$ $\left(3 \times 10^{-4}\right.$ Earth masses) for $q=5$. For comparison, the mass of the asteroid belt is estimated ${ }^{25,26}$ to be $3 \times 10^{21} \mathrm{~kg}$.
Figures 1-3 illustrate that, if the $24-27$ AU belt is shown to be populated, material would still be escaping from this region. Just as the Kuiper belt serves as a reservoir and source of material, the bodies in the 24-27 AU region that develop Uranus- or Neptunecrossing orbits could contribute to the flux of ecliptic comets in the outer planet region. The steady-state population of such comets originating in the $24-27 \mathrm{AU}$ belt is roughly

$$
N_{\mathrm{c}, \text { ind }} \approx \frac{\tau_{\mathrm{c}}}{\tau_{\text {ind }}} N_{\text {ind }}
$$

where $\tau_{\mathrm{c}}$ is the dynamical lifetime of a comet leaving the $24-27 \mathrm{AU}$ region and $\tau_{\text {ind }}$ is the dynamical lifetime of a body in the $24-27 \mathrm{AU}$ region before it develops a planet-crossing orbit. If we assume that the lifetime of a comet originating in the 24-27 AU belt is similar to that of a comet from the Kuiper belt, then ${ }^{9} \tau_{\mathrm{c}}=4.5 \times 10^{7} \mathrm{yr}$. From the $t^{-1}$ decay of the population in the $24-26 \mathrm{AU}$ belt, we get $\tau_{\text {ind }} \approx 4.5 \times 10^{9} \mathrm{yr}$. These values yield

$$
N_{\mathrm{c} \text {,ind }} \approx 10^{-2} N_{\text {ind }}=4 \times 10^{-5} N_{\mathrm{k}}
$$

For comparison the steady-state number of ecliptic comets originating in the Kuiper belt is roughly

$$
N_{\mathrm{c}, \mathrm{k}} \approx \frac{\tau_{\mathrm{c}}}{\tau_{\mathrm{k}}} N_{\mathrm{k}}=2 \times 10^{-3} N_{\mathrm{k}}
$$

where $\tau_{\mathrm{k}}=2 \times 10^{10} \mathrm{yr}$ is the typical dynamical lifetime of a Kuiperbelt object before it encounters Neptune and $\tau_{\mathrm{c}}=4.5 \times 10^{7} \mathrm{yr}$ as before ${ }^{9,21}$. By this comparison, the $24-27 \mathrm{AU}$ belt adds $2 \%$ to the steady-state number of Centaurs and short-period comets in the outerplanet region. Caution should be exercised here as all the numbers used are uncertain.

The simple calculations presented here open a number of avenues for future research. First, other dynamical processes such as collisions or outer-planet migration ${ }^{17-20}$ could be explored to assess their effect on the distribution of surviving objects in the 24-27 $\mathrm{AU}$ region. Second, the $\mathrm{L}_{4}$ and $\mathrm{L}_{5}$ points of the outer planets, as well as other regions that could potentially harbour residual mass, should be carefully considered. Third, surveys with fainter limiting magnitudes and more extensive sky-plane coverage will either reveal members of the 24-27 Au belt or place more significant limits on the population in the region. A survey of 8 square degrees to $m_{R}=26.2$, sensitive to objects with radii $z>10 \mathrm{~km}$ at $25 \mathrm{AU}$ assuming $p_{R}=0.04$, would reduce the current upper limit on $N(>10 \mathrm{~km})$ by an order of magnitude. Although such a survey would be a tremendous undertaking with today's technology, it could be more easily accomplished by using telescopes with larger collecting areas and larger fields of view; such telescopes should soon become available. The discovery of material in the $24-27 \mathrm{AU}$ region would imply either that the processes occuring during planet formation left the material in this region undisturbed or that some other process later repopulated the region. The absence of members of the 24-27 AU belt would imply the occurrence of some event, such as planet migration, that disrupted this fragile population.

Received 2 December 1996; accepted 16 April 1997.

1. Jewitt, D. \& Luu, J. X. Discovery of the candidate Kuiper belt object 1992QB 1 . Nature 362, 730-732 (1993).

2. Irwin, M., Tremaine, S. \& Zytkow, A. N. A search for slow-moving objects and the luminosity function of the Kuiper belt. Astron. J. 110, 3082-3092 (1995).

3. Williams, I. P., O'Ceallaigh, D. P., Fitzsimmons, A. \& Marsden, B. G. The slow-moving objects 1993SB and 1993SC. Icarus 116, 180-185 (1995).

4. Jewitt, D., Luu, J. \& Chen, J. The Mauna Kea-Cerro-Tololo (MKCT) Kuiper belt and Centaur survey. Astron. J. 112, 1225-1238 (1996).

5. Cochran, A. L., Levison, H. F., Stern, S. A. \& Duncan, M. J. The discovery of Halley-sized Kuiper belt objects using the Hubble Space Telescope. Astrophys. J. 455, 342-346 (1995).

6. Duncan, M., Quinn, T. \& Tremaine, S. Astrophys. J. 328, L69-L73 (1988).

7. Holman, M. J. \& Wisdom, J. Dynamical stability in the outer solar system and the delivery of short period comets. Astron. J. 105, 1987-1999 (1993).

8. Levison, H. F. \& Duncan, M. J. The gravitational sculpting of the Kuiper belt. Astrophys. J. 406, L35L38 (1993).

9. Levison, H. F. \& Duncan, M. J. From the Kuiper belt to Jupiter-family comets: the spatial distribution of ecliptic comets. Icarus (in the press).

10. Gladman, B. \& Duncan, M. J. On the fates of minor bodies in the outer solar system. Astron. J. 100, $1680-1693$ (1990). 
11. Holman, M. in Proc. 27th Symp. on Celestial Mechanics (eds Kinoshita, H. \& Nakai, H.) 116-136 (Nat Astron. Observatory of Japan, Tokyo, 1995).

12. Roy, A. E. Orbital Motion (Hilger, Bristol, 1988).

13. Levison, H. F. \& Duncan, M. J. The long-term dynamical behavior of short-period comets. Icarus 108, 18-36 (1994).

14. Dones, L., Levison, H. F. \& Duncan, M. Completing the Inventory of the Solar System (eds Rettig, T. W. \& Hahn, J. M.) 233-244 (ASP Conf. Proc., Astron. Soc. Pacif., San Francisco, 1996).

15. Stern, S. A. Collision timescales in the Kuiper disk: model estimates and their implications. Astron. J. 110, 856-868 (1995)

16. Davis, D. R. \& Farinella, P. Collisional evolution of Edgeworth-Kuiper belt objects. Icarus 125, 50-60 (1997).

17. Fernandez, J. A. \& Ip, W. H. Some dynamical aspects of the accretion of Uranus and Neptune: the exchange of orbital angular momentum with planetesimals. Icarus 58, 109-120 (1984).

18. Malhotra, R. The origin of Pluto's peculiar orbit. Nature 365, 819-821 (1993).

19. Malhotra, R. The origin of Pluto's orbit: implications for the solar system beyond Neptune. Astron J. 110, 420-429 (1995)

20. Morbidelli, A. \& Valsecchi, G. B. Neptune scattered planetesimals could have sculpted the primordial Edgeworth-Kuiper belt. Icarus (in the press).

21. Duncan, M. J., Levison, H. F. \& Budd, S. M. The dynamical structure of the Kuiper belt. Astron. J. 110, 3073-3081 (1996)

22. Tremaine, S. in Baryonic Dark Matter (eds Lynden-Bell, D. \& Gilmore, G.) 37-65 (Kluwer, Boston, 1990)

23. Kowal, C. T. A solar system survey. Icarus 77, 118-123 (1989).

24. Weissman, P. R. \& Levison, H. F. The size distribution of cometary nuclei. Lunar Planet. Sci. 27, 1409 (1996).

25. Kresak, L. Mass content and mass distribution of the asteroid system. Bull. Astron. Inst. Czech. 28, 65 $82(1977)$

26. Morrison, D. Asteroid sizes and albedos. Icarus 31, 185-220 (1977).

Acknowledgements. I thank N. Murray and S. Tremaine for discussions, S. Dermott and H. Levison fo reviews of the manuscript, and J. Wisdom for computer time for the simulations.

\section{Cluster-derived structures and} conductance fluctuations in

\section{nanowires}

\section{R. N. Barnett \& Uzi Landman}

School of Physics, Georgia Institute of Technology, Atlanta, Georgia 30332, USA

Understanding the variation of a material's properties with size, form of aggregation and dimensionality is becoming important in the face of increasing miniaturization of electronic and mechanical devices. Experimental studies have focused on the preparation and characterization of solid-state nanometre-scale structures such as metal and semiconductor nanocrystals ${ }^{1-3}$, surface-supported structures and quantum $\operatorname{dots}^{4}$ and nanoscale junctions or wires $^{5-22}$. It has emerged that these nanostructures can often be fruitfully described using concepts and methodologies developed in the contexts of gas-phase atomic clusters and atomic nuclei ${ }^{23-25}$. Here we make this connection explicitly through first-principle molecular dynamics simulations ${ }^{22,26}$ which show that, as nanowires of sodium metal are stretched to just a few atoms in diameter, the structures formed by metal atoms in the neck can be described in terms of those observed in small gas-phase sodium clusters $^{27}$. We find that the electronic spectral and conductance characteristics of these atomic-scale contacts exhibit dynamical thermal fluctuations on a sub-picosecond timescale, owing to rearrangements of the metal atoms, which will significantly affect the transport properties of such nanowires.

Formation and mechanical properties of interfacial junctions (in the form of nanowires) have been predicted through early molecular dynamics simulations ${ }^{5}$, where the materials were modelled using semiempirical embedded-atom potentials. In these studies it was shown that separation of the contact between materials leads to generation of a connective junction which elongates and narrows through a sequence of structural instabilities; at the early stages elongation of the junction involves multiple slip events, whereas at the later stages, when the lateral dimension of the wire narrows to a diameter of $\sim 15 \AA$, further elongation involves a succession of stress accumulation and fast relief stages associated with a sequence of order-disorder structural transformations localized to the neck region ${ }^{5,19,20}$. These predictions, as well as anticipated electronic conductance properties ${ }^{5,6}$ have been corroborated in a number of experiments using scanning tunnelling and force microscopy $^{5,7-9,11,16,21}$, break junctions ${ }^{10}$ and pin-plate techniques $^{13,19}$ in ambient environments, as well as under ultra-high vacuum and/or cryogenic conditions.

One of the most fascinating aspects of such nanowires is the prospect of formation of atomic-scale contacts and switches, $5,11,14,18$ which may occur towards the ultimate stages of elongation (that is before complete physical separation, or breaking, of the wire). Understanding the atomic rearrangements and structural evolution, electronic properties, and dynamics of such nanojunctions, particularly as they approach the 'one-atom contact' regime, is a significant challenge requiring a detailed theoretical description based on first-principles electronic structure calculations. To this end we have used the Born-Oppenheimer (BO) local-spin-density (LSD) molecular dynamics (MD) method (for details of the BOLSD-MD, see ref. 26) for sodium wires, where the dynamics of the ions (with an integration time-step of $3 \mathrm{fs}$ ) evolves at finite temperature $(T=190 \mathrm{~K})$ on concurrently calculated ground-state potential-energy surfaces, evaluated self-consistently for each ionic configuration using the LSD theory, in conjunction with non-local norm-conserving pseudopotentials ${ }^{28}$ with a $6.2 \mathrm{Ry}$ plane-wave energy cut-off.

The simulation starts from a sodium block of bulk body-centred cubic (b.c.c.) (100) layers oriented along the $z$-axis and tapered to a narrowing in the middle. The periodic super-cell with $(x, y, z)$ dimensions $(19.98 \AA, 19.98 \AA, 21.3 \AA$ ) contains a total of 119 atoms treated dynamically, supported at the top and bottom by static (100) layers (25 atoms each); one of these layers is in the cell and the other is its periodic image. After equilibration the structure of the junction is mostly b.c.c., except in the middle layer (Fig. 1a). Each subsequent elongation involves a uniform dilation in the $z$ direction, followed by steepest-descent energy minimization and dynamical equilibration for several picoseconds (at the start of the equilibration period stochastic thermalization to $190 \mathrm{~K}$ is used).

The atomic configurations of the sodium wire at selected stages of elongation shown in Fig. 1 reveal development of cluster-derived structures at the narrow-neck region. Particularly striking is the formation of a 13-atom (slightly distorted) icosahedron (5-fold symmetric), supported between the upper and lower parts of the wire (Fig. 1b,c); this structure may also be viewed as two $\mathrm{Na}_{7}$ pentagonal bipyramidal clusters sharing an apex atom. Subsequent elongation results in 'opening' of the structure (Fig. 1d), and stretching of the contact past this stage results in formation of a pentagonal bipyramidal $\mathrm{Na}_{7}$ cluster (top) bonded through one of its apex atoms to the upper atom of a tetrahedral cluster (bottom), giving the appearance of a stretched sodium dimer at the narrowing (Fig. 1e). Further elongation results in breaking of the nanowire (Fig. 1f). The occurrence of these molecular-like supported structures, which correlate with those derived for isolated sodium clusters $^{27}$, suggests that in atomic-scale narrow contacts the atomic coordination and nature of bonding may be described using information from investigations pertaining to small (isolated) clusters. In a certain sense the reduced dimensions, increased surface-to-volume ratio, and atomically impoverished environment in such nanoscale solid-state junctions provide a link to the realm of finite-size cluster science, where ground-state (and isomeric) structures are different from the bulk, and 'magic numbers' are known to occur $^{23-25,27}$. (Here 'magic numbers' refers to the enhanced stability of certain sequences of cluster sizes, shapes and structural motifs due to electronic and/or geometric-packing shell effects, which may vary from one class of materials to another.)

Contour plots of the self-consistent local effective potential confining the electrons to the wire are shown in Fig. 2 for the 23.97- $\AA$ and $29.96-\AA$ wire configurations (compare Fig. $1 \mathrm{~b}$ and e). 